\title{
CRISPR-Cas regulation: a systems biology approach
}

\author{
M.J. Djordjevic ${ }^{*}$, A. Rodic ${ }^{1}$, B. Blagojevic ${ }^{2}$, M.R. Djordjevic ${ }^{2}$, K. Severinov ${ }^{3}$ \\ ${ }^{1}$ Faculty of Biology, Institute of Physiology and Biochemistry, University of Belgrade, Serbia \\ ${ }^{2}$ Institute of Physics Belgrade, University of Belgrade, Serbia \\ ${ }^{3}$ Skolkovo Institute of Science and Technology, Moscow, Russia \\ *e-mail:dmarko@bio.bg.ac.rs
}

Key words: computational systems biology, CRISPR-Cas, small RNAs, gene expression regulation

Motivation and Aim: Bacterial immune systems (CRISPR-Cas and restrictionmodification systems) defend bacterial cells against invasion by viruses or plasmids. CRISPR array is transcribed as a long transcript (pre-crRNA), which is processed by Cas proteins to small interfering RNAs (crRNAs). CRISPR-Cas is typically silent under normal conditions, and one of the main questions in understanding CRISPR-Cas functioning is how this normally silent system is induced. Previous work (by us and others) shows that the system is regulated both at transcriptional and post-transcriptional (pre-crRNA processing) level.

Approach and Methods: To understand mechanism of CRISPR-Cas induction, we use a systems biology approach, combining computational modeling with bioichemical experiments, and first available in-vivo measurements of molecule dynamics in bacterial immune systems (done by Konstantin Severinov lab, Skoltech). Moreover, we exploit that CRISPR-Cas and more rudimentary restriction-modification (R-M) systems, likely exhibit similar constraints in their dynamical response, so that modeling better characterized R-M systems can aid understanding CRISPR-Cas. Computational modeling of CRISPR-Cas and R-M system induction/establishment is based on thermodynamical modeling of transcription regulation, and on modeling dynamics of the relevant molecular species (RNA and proteins). Wild-type systems are in-silico perturbed to assess how key regulatory features contribute to their dynamics.

Results: We show that computational modeling can reasonably explain both in-vitro measurements of transcription regulation, and in-vivo measurements of protein dynamics in R-M systems. We furthermore explore R-M systems with different architectures, and find that their (otherwise diverse) regulation can be explained in terms of few simple design principles. For CRISPR-Cas we show that both of the key system features (high cooperativity in transcription regulation and fast pre-crRNA processing) are responsible for a fast (switch-like) transition of the system from "OFF" to "ON" state, which we also obtain for R-M systems, and which can be explained in terms of efficiently protecting the host cell. Additionally, fast pre-crRNA processing also leads to a delay in crRNA generation, similarly to the delay in restriction enzyme synthesis during R-M establishment, and which may be related with evading auto-immune response. Finally, we find that the cooperative transcription regulation qualitatively leads to a cross-over to the regime where at higher pre-crRNA processing rates crRNA generation approaches the limit of (an infinitely abrupt) system induction.

Conclusion: We propose that few simple design principles may be behind regulation of mechanistically diverse bacterial immune systems, which is likely related with a highly efficient host cell protection, while at the same time evading autoimmunity. Acknowledgement: Support by MESTD of Serbia OI173052 and SNSF IZ73Z0_152297. 